\title{
Dynamics of complex singularities in 1D nonlinear parabolic PDE's
}

\author{
by \\ Zoran Grujić (Austin, TX and Charlottesville, VA)
}

\begin{abstract}
We establish local-in-time smoothing of a simple model nonlinear parabolic PDE in a scale of weighted Bergman spaces on a strip provided the weights are not too singular. This constitutes a very strong smoothing property since an immediate consequence is that the PDE can "push away" an algebraic-type complex singularity provided that the order of the singularity is small enough.
\end{abstract}

1. Introduction. Local-in-time analytic smoothing of nonlinear parabolic PDE's with analytic nonlinearities is a well known phenomenon. Roughly speaking, the same type of initial data that guarantees local-in-time existence of a regular solution also guarantees local-in-time spatial analyticity. An elegant method of explicitly estimating the uniform analyticity radius of solutions on periodic domains in terms of the $L^{2}$-based Sobolev norms of the initial data, the Gevrey class method, was introduced in [FT] for the Navier-Stokes equations, and has since had numerous generalizations and applications (see e.g. [CEES, FeTi]). A related method was presented in [GK1, GK2], providing analogous results in terms of $L^{p}$-norms of the initial data, and also dealing with the issue of estimating the analyticity radius of solutions on bounded domains.

We focus on the case of one space dimension where the original spatial domain is equal to the whole real line. Consider, for simplicity, a model nonlinear parabolic PDE with a power-type nonlinearity, $(\partial / \partial t) u+\left(-\partial^{2} / \partial z^{2}\right)^{m} u$ $=u^{n}$, posed in $L^{2}$-type spaces. The aforementioned methods also provide local-in-time smoothing in a scale of analytic Gevrey spaces, or equivalently, $L^{2}$-type Hardy spaces on a complex strip. More precisely, if the inital datum is in $H^{2}\left(S_{\tau}\right)$, i.e., in a classical Hardy space on a complex strip

$$
S_{\tau}=\{z \in \mathbb{C}:|\Im z|<\tau\},
$$

and if $n \leq n^{*}(m)$, then there exists $T\left(\left\|u_{0}\right\|_{H^{2}\left(S_{\tau}\right)}, m, n\right)>0$ such that $u(t) \in H^{2}\left(S_{\tau+(1 / c) t^{1 /(2 m)}}\right)$ for all $t \in[0, T]$.

2000 Mathematics Subject Classification: 30H05, 35B65. 
It is of great interest to study the dynamics of singularities of a solution in the complex plane. In particular, finite-time blow-up of a solution at some time $T$ can be detected by loss of analyticity at $T$, and one of the possible scenarios is that the loss of analyticity is caused by complex singularities migrating to the real axis. A step in the direction of establishing such results would be to work in a scale of spaces that allow a certain algebraic rate of blow-up of integral means

$$
M_{p}(y ; u)=\left(\int_{-\infty}^{\infty}|u(x+i y)|^{p} d x\right)^{1 / p}
$$

as $y \rightarrow \tau$. (Recall that, in contrast, $u \in H^{p}\left(S_{\tau}\right)$ if $\lim _{y \rightarrow \tau} M_{p}(y ; u)$ is finite.) Inital data with such a property are interesting because an algebraic-type singularity at $\Im z=\tau$ would generally correspond to an algebraic rate of blow-up, $M_{p}(y ; u) \sim 1 /(\tau-y)^{\alpha}$ for some $\alpha>0$. The goal of this paper is to indicate that weighted Bergman spaces on a strip $B_{\alpha}^{p}\left(S_{\tau}\right)$ may provide a suitable basis for carrying out a program using these ideas.

In Section 2, we define the spaces and prove some inequalities including $L^{\infty}$-interpolation that will be used to handle a nonlinear term in our simple model PDE. In Section 3 we prove the main result - local-in-time smoothing in a scale of weighted Bergman spaces on a strip.

2. Weighted Bergman spaces on a strip. For $\Omega \subseteq \mathbb{C}$, denote by $A(\Omega)$ the set of $\mathbb{C}$-valued functions analytic on $\Omega$. Also, let $\mathbb{D}=\{z \in \mathbb{C}$ : $|z|<1\}$ be the open unit disc.

Let $f \in A(\mathbb{D})$. The study of integral means

$$
M_{p}(r ; f)=\left(\frac{1}{2 \pi} \int_{0}^{2 \pi}\left|f\left(r e^{i \varphi}\right)\right|^{p} d \varphi\right)^{1 / p} \quad \text { as } r \rightarrow 1
$$

$(0<p<\infty)$ is a classical theory founded by Hardy and Littlewood in the 1930s. A self-contained survey including many historical references can be found in $[\mathrm{D}]$. Recall that Hardy $H^{p}$-spaces consist of functions $f \in A(\mathbb{D})$ for which

$$
\lim _{r \rightarrow 1} M_{p}(r ; f)<\infty,
$$

and Bergman $B^{p}$-spaces are those functions $f \in A(\mathbb{D})$ for which

$$
\int_{0}^{1} M_{p}^{p}(r ; f) d r<\infty .
$$

Weighted Bergman spaces $B_{\alpha}^{p}, 0<p<\infty, \alpha>-1$ are spaces of functions $f \in A(\mathbb{D})$ for which 


$$
\int_{0}^{1}(1-r)^{\alpha} M_{p}^{p}(r ; f) d r<\infty .
$$

For $1 \leq p<\infty$, the $B_{\alpha}^{p}$ are Banach spaces with norms

$$
\|f\|_{B_{\alpha}^{p}}=\left(\int_{0}^{1}(1-r)^{\alpha} M_{p}^{p}(r ; f) d r\right)^{1 / p} .
$$

For some properties of $B_{\alpha}^{p}$ see [NOW].

The theory in $\mathbb{D}$ has an analog on a complex strip $S_{\tau}$, where the integral means

$$
M_{p}(y ; u)=\left(\int_{-\infty}^{\infty}|u(x+i y)|^{p} d x\right)^{1 / p}
$$

are studied as $y \rightarrow \tau$.

Henceforth, assume $u(\mathbb{R}) \subseteq \mathbb{R}$ so that we can confine our considerations to the half-plane $\{z \in \mathbb{C}: \Im z \geq 0\}$.

Let $1 \leq p<\infty, \alpha>-1$. Then the analogs of the $B_{\alpha}^{p}$ are the Banach spaces

$$
\begin{aligned}
B_{\alpha}^{p}\left(S_{\tau}\right)=\left\{u \in A\left(S_{\tau}\right): u(\mathbb{R}) \subseteq \mathbb{R}\right. & \\
\|u\|_{B_{\alpha}^{p}\left(S_{\tau}\right)} & \left.=\left(\int_{0}^{\tau}(\tau-y)^{\alpha} M_{p}^{p}(y ; u) d y\right)^{1 / p}<\infty\right\} .
\end{aligned}
$$

The goal of this section is to state and prove two basic inequalities that will enable us to obtain energy-type estimates in $B_{\alpha}^{2}\left(S_{\tau}\right)$. The first one shows how to trade weights for derivatives.

Proposition 1. Let $u \in A\left(S_{\tau}\right)$ for some $\tau>0, u(\mathbb{R}) \subseteq \mathbb{R}$, and $\alpha>-1$. Then

$$
\begin{aligned}
& \int_{0}^{\tau}(\tau-y)^{\alpha} \int_{-\infty}^{\infty}|u(x+i y)|^{2} d x d y \\
& \leq \frac{2 \tau^{\alpha+1}}{\alpha+1} \int_{-\infty}^{\infty}|u(x+i 0)|^{2} d x \\
& \quad+\frac{4}{(\alpha+1)^{2}} \int_{0}^{\tau}(\tau-y)^{\alpha+2} \int_{-\infty}^{\infty}\left|u^{\prime}(x+i y)\right|^{2} d x d y
\end{aligned}
$$

Proof. Assume first that $u$ is analytic on the closed strip. Integrating by parts gives 


$$
\begin{aligned}
& \int_{0}^{\tau}(\tau-y)^{\alpha} \int_{-\infty}^{\infty}|u(x+i y)|^{2} d x d y \\
&=\frac{\tau^{\alpha+1}}{\alpha+1} \int_{-\infty}^{\infty}|u(x+i 0)|^{2} d x \\
&-\frac{1}{\alpha+1} \int_{0}^{\tau}(\tau-y)^{\alpha+1} \frac{\partial}{\partial y} \int_{-\infty}^{\infty}|u(x+i y)|^{2} d x d y
\end{aligned}
$$

Since

$$
\left.\left|\frac{\partial}{\partial y} \int_{-\infty}^{\infty}\right| u(x+i y)\right|^{2} d x\left|\leq 2 \int_{-\infty}^{\infty}\right| u(x+i y)|\cdot| u^{\prime}(x+i y) \mid d x,
$$

the last term in (2) can be estimated by

$$
\begin{aligned}
\frac{2}{\alpha+1} \int_{0}^{\tau}(\tau-y)^{\alpha+1}( & \left.\int_{-\infty}^{\infty}|u(x+i y)|^{2} d x\right)^{1 / 2} \\
& \times\left(\int_{-\infty}^{\infty}\left|u^{\prime}(x+i y)\right|^{2} d x\right)^{1 / 2} d y \\
\leq & \frac{2}{\alpha+1}\left(\int_{0}^{\tau}(\tau-y)^{\alpha} \int_{-\infty}^{\infty}|u(x+i y)|^{2} d x d y\right)^{1 / 2} \\
& \times\left(\int_{0}^{\tau}(\tau-y)^{\alpha+2} \int_{-\infty}^{\infty}\left|u^{\prime}(x+i y)\right|^{2} d x d y\right)^{1 / 2} \\
\leq & \frac{1}{2} \int_{0}^{\tau}(\tau-y)^{\alpha} \int_{-\infty}^{\infty}|u(x+i y)|^{2} d x d y \\
& +\frac{2}{(\alpha+1)^{2}} \int_{0}^{\tau}(\tau-y)^{\alpha+2} \int_{-\infty}^{\infty}\left|u^{\prime}(x+i y)\right|^{2} d x d y
\end{aligned}
$$

Inserting (3) in (2) yields the desired inequality.

If $u$ is analytic only on an open strip, then we can apply the above argument to $\{z \in \mathbb{C}:|\Im z|<\tau-\varepsilon\}$, and then pass to the limit utilizing the Lebesgue Monotone Convergence Theorem.

REMARK 1. The proof is an analog of the proof of the corresponding result for $\mathbb{D}$ (see Theorem 5.6 of $[\mathrm{D}]$ ). The only difference is that integration by parts in radial direction is replaced by integration in $y$-direction, and hence $|f(0)|$ is replaced by the integral of $|u|$ along the real axis. 
Proposition 2. Let $u \in A\left(S_{\tau}\right)$ for some $\tau>0, u(\mathbb{R}) \subseteq \mathbb{R}$, and $\alpha>0$. Then

$$
\begin{aligned}
|u(x+i y)| \leq & \frac{c}{(\tau-y)^{1 / 2}}\left(\int_{-\infty}^{\infty}|u(x+i 0)|^{2} d x\right)^{1 / 2} \\
& +\frac{c_{\alpha}}{(\tau-y)^{(\alpha+1) / 2}}\left(\int_{0}^{\tau}(\tau-y)^{\alpha} \int_{-\infty}^{\infty}|u(x+i y)|^{2} d x d y\right)^{1 / 4} \\
& \times\left(\int_{0}^{\tau}(\tau-y)^{\alpha} \int_{-\infty}^{\infty}\left|u^{\prime}(x+i y)\right|^{2} d x d y\right)^{1 / 4}
\end{aligned}
$$

for all $x \in \mathbb{R}$ and $0<y<\tau$.

We will split the proof of Proposition 2 in three lemmas.

Lemma 1. Let $u \in A\left(S_{\tau}\right)$ for some $\tau>0, u(\mathbb{R}) \subseteq \mathbb{R}$, and $\alpha>-1$. Then

$$
\int_{-\infty}^{\infty}|u(x+i y)| d x \leq \frac{2^{\alpha+1}}{(\tau-y)^{\alpha+1}} \int_{0}^{\tau}(\tau-s)^{\alpha} \int_{-\infty}^{\infty}|u(x+i s)| d x d s
$$

for all $0<y<\tau$.

Proof. Let $0<y<\tau$, and consider a closed interval $[y,(y+\tau) / 2]$. Then

$$
\begin{aligned}
\int_{0}^{\tau}(\tau-s)^{\alpha} \int_{-\infty}^{\infty}|u(x+i s)| d x d s & \geq \int_{y}^{(y+\tau) / 2}(\tau-s)^{\alpha} \int_{-\infty}^{\infty}|u(x+i s)| d x d s \\
& =\frac{\tau-y}{2}(\tau-\eta)^{\alpha} \int_{-\infty}^{\infty}|u(x+i \eta)| d x
\end{aligned}
$$

for some $\eta \in[y,(y+\tau) / 2]$ by the Mean Value Theorem. Since $\tau-\eta \geq$ $(\tau-y) / 2$, the last line in (4) is bounded below by

$$
\frac{(\tau-y)^{\alpha+1}}{2^{\alpha+1}} \int_{-\infty}^{\infty}|u(x+i \eta)| d x
$$

and since the integral means are monotone in $s$,

$$
\int_{-\infty}^{\infty}|u(x+i \eta)| d x \geq \int_{-\infty}^{\infty}|u(x+i y)| d x
$$

concluding the proof.

Lemma 2. Let $u \in A\left(S_{\tau}\right)$ for some $\tau>0, u(\mathbb{R}) \subseteq \mathbb{R}$, and $\alpha>0$. Then

$$
\begin{aligned}
\int_{-\infty}^{\infty}|u(x+i y)| d x \leq & \int_{-\infty}^{\infty}|u(x+i 0)| d x \\
& +\frac{2^{\alpha+1}}{\alpha} \cdot \frac{1}{(\tau-y)^{\alpha}} \int_{0}^{\tau}(\tau-s)^{\alpha} \int_{-\infty}^{\infty}\left|u^{\prime}(x+i s)\right| d x d s
\end{aligned}
$$

for all $0<y<\tau$. 
Proof. Let $0<y<\tau$. Then

$$
|u(x+i y)| \leq|u(x+i 0)|+\int_{0}^{y}\left|u^{\prime}(x+i s)\right| d s .
$$

Integrating (7) in $x$ gives

$$
\int_{-\infty}^{\infty}|u(x+i y)| d x \leq \int_{-\infty}^{\infty}|u(x+i 0)| d x+\int_{-\infty}^{\infty} \int_{0}^{y}\left|u^{\prime}(x+i s)\right| d s d x
$$

Utilizing the Fubini Theorem, and integrating the inequality in Lemma 1 applied to $u^{\prime}$, we obtain the estimate.

Lemma 3. Let $u \in A\left(S_{\tau}\right)$ for some $\tau>0, u(\mathbb{R}) \subseteq \mathbb{R}$, and $\alpha>0$. Then

$$
\begin{aligned}
|u(x+i y)| \leq & \frac{2}{\pi} \cdot \frac{1}{\tau-y} \int_{-\infty}^{\infty}|u(x+i 0)| d x \\
& +\frac{2^{2(\alpha+1)}}{\pi \alpha} \cdot \frac{1}{(\tau-y)^{\alpha+1}} \int_{0}^{\tau}(\tau-s)^{\alpha} \int_{-\infty}^{\infty}\left|u^{\prime}(x+i s)\right| d x d s
\end{aligned}
$$

for all $x \in \mathbb{R}$ and $0<y<\tau$.

Proof. Let $x \in \mathbb{R}, 0<y<\tau$. Then, by the Cauchy formula,

$$
\begin{aligned}
|u(x+i y)| \leq & \frac{1}{2 \pi} \int_{\Im \zeta=y+(\tau-y) / 2} \frac{|u(\zeta)|}{|\zeta-(x+i y)|}|d \zeta| \\
& +\frac{1}{2 \pi} \int_{\Im \zeta=y-(\tau-y) / 2} \frac{|u(\zeta)|}{|\zeta-(x+i y)|}|d \zeta| .
\end{aligned}
$$

Since $|\zeta-(x+i y)| \geq(\tau-y) / 2$, the right-hand side of $(8)$ is bounded by

$$
\begin{aligned}
\frac{1}{\pi} \frac{1}{\tau-y} \int_{-\infty}^{\infty} \mid u(x+i(y+(\tau-y) / 2) \mid d x & \\
& +\frac{1}{\pi} \frac{1}{\tau-y} \int_{-\infty}^{\infty} \mid u(x+i(y-(\tau-y) / 2) \mid d x,
\end{aligned}
$$

which is by the monotonicity of integral means (and conjugate symmetry if $y<\tau / 3)$ bounded by

$$
\frac{2}{\pi} \cdot \frac{1}{\tau-y} \int_{-\infty}^{\infty} \mid u(x+i(y+(\tau-y) / 2) \mid d x .
$$

Estimating the integral in (9) by Lemma 2 finishes the proof.

Proof of Proposition 2. Apply Lemma 3 to $u^{2}$, estimate the area integral by the Cauchy-Schwarz inequality, and take the square root.

Remark 2. Proposition 2 is an Agmon-type interpolation inequality in a $B_{\alpha}^{2}$-setting. 


\section{A nonlinear parabolic PDE in a scale of weighted Bergman} spaces. For simplicity of exposition we consider $L^{2}$-type spaces $B_{\alpha}^{2}\left(S_{\tau}\right)$. (The interpolation inequalities in Propositions 1 and 2 are in $B_{\alpha}^{2}\left(S_{\tau}\right)$-setting; however, the corresponding inequalities in the general $B_{\alpha}^{p}\left(S_{\tau}\right)$-setting can be obtained using similar techniques.)

Also, since our primary goal is to show that one can derive energy-type inequalities in the setting of weighted Bergman spaces, we will not always strive to obtain an optimal estimate.

Consider a very simple model nonlinear parabolic PDE with a quadratic nonlinearity $(n=m=2)$,

$$
u_{t}+u_{z z z z}=u^{2}
$$

in a scale of weighted Bergman spaces $B_{\alpha}^{2}\left(S_{\tau}\right), \tau \geq \tau_{0}>0$, where $u_{0}=$ $u(0) \in B_{\alpha}^{2}\left(S_{\tau_{0}}\right)$. (It is expected that higher (relative to the positive linear part) nonlinearities can be treated by working in $B_{\alpha}^{p}\left(S_{\tau}\right)$, for $p$ large enough.)

Notice that applying Lemma 1 to $u_{0}^{2}$, and letting $y \rightarrow 0$, we obtain

$$
\left\|u_{0}\right\|_{L^{2}(\mathbb{R})} \leq c_{\tau_{0}, \alpha}\left\|u_{0}\right\|_{B_{\alpha}^{2}\left(S_{\tau_{0}}\right)}<\infty .
$$

Consequently, using standard techniques, one can show that there exists $T^{*}\left(\left\|u_{0}\right\|_{B_{\alpha}^{2}\left(S_{\tau_{0}}\right)}, \tau_{0}, \alpha\right)>0$ such that

$$
\|u(t)\|_{L^{2}(\mathbb{R})} \leq c_{\tau_{0}, \alpha}\left\|u_{0}\right\|_{B_{\alpha}^{2}\left(S_{\tau_{0}}\right)}
$$

for all $t \in\left[0, T^{*}\right]$. The inequality (12) provides the control of the trace of a complexified solution on the real axis that is needed in the inequalities in Section 2.

We are now ready to state our main result.

TheOREM 1. Let $u_{0} \in B_{\alpha}^{2}\left(S_{\tau_{0}}\right)$ for some $\tau_{0}>0, \alpha \leq 3$. Then there exists $T\left(\left\|u_{0}\right\|_{B_{\alpha}^{2}\left(S_{\tau_{0}}\right)}, \tau_{0}, \alpha\right)>0$ such that $u(t) \in B_{\alpha}^{2}\left(S_{\tau_{0}+t}\right)$ for all $t \in[0, T]$.

REMARK 3. Considering the expansion of the analyticity radius $\gamma(t)=$ $\tau_{0}+\delta t$, and then optimizing in $\delta$, one finds that $u(t) \in B_{\alpha}^{2}\left(S_{\tau_{0}+(1 / c) t^{1 / 4}}\right)$, which is favorable for small $t$, and also obeys the correct space-time scaling.

Proof of Theorem 1. For $t \geq 0$, consider

$$
\varphi(t)=\int_{0}^{\tau_{0}+t}\left(\left(\tau_{0}+t\right)-y\right)^{\alpha} \int_{-\infty}^{\infty}|u(x+i y, t)|^{2} d x d y .
$$

Notice that $\varphi(0)=\left\|u_{0}\right\|_{B_{\alpha}^{2}\left(S_{\tau_{0}}\right)}^{2}<\infty$. Also,

$$
\begin{aligned}
\frac{d}{d t} \varphi(t)= & \alpha \int_{0}^{\tau_{0}+t}\left(\left(\tau_{0}+t\right)-y\right)^{\alpha-1} \int_{-\infty}^{\infty}|u(x+i y, t)|^{2} d x d y \\
& +\int_{0}^{\tau_{0}+t}\left(\left(\tau_{0}+t\right)-y\right)^{\alpha} \frac{\partial}{\partial t} \int_{-\infty}^{\infty}|u(x+i y, t)|^{2} d x d y
\end{aligned}
$$


To obtain a differential inequality for $\varphi$, we multiply (10) by $\bar{u}$, take the real part, integrate in $x$, multiply by $\left(\left(\tau_{0}+t\right)-y\right)^{\alpha}$, and integrate in $y$. Integration by parts (in $x$ ) yields

$$
\begin{aligned}
\frac{1}{2} \int_{0}^{\tau_{0}+t}\left(\left(\tau_{0}+t\right)-y\right)^{\alpha} & \frac{\partial}{\partial t} \int_{-\infty}^{\infty}|u(x+i y, t)|^{2} d x d y \\
& +\int_{0}^{\tau_{0}+t}\left(\left(\tau_{0}+t\right)-y\right)^{\alpha} \int_{-\infty}^{\infty}\left|u^{\prime \prime}(x+i y, t)\right|^{2} d x d y \\
\leq & \int_{0}^{\tau_{0}+t}\left(\left(\tau_{0}+t\right)-y\right)^{\alpha} \int_{-\infty}^{\infty}|u(x+i y, t)|^{3} d x d y
\end{aligned}
$$

$\left(u^{\prime}=u_{x}=u_{z}\right)$. Utilizing (14) gives

$$
\begin{aligned}
\frac{d}{d t} \int_{0}^{\tau_{0}+t}\left(\left(\tau_{0}+t\right)-y\right)^{\alpha} & \int_{-\infty}^{\infty}|u(x+i y, t)|^{2} d x d y \\
& +2 \int_{0}^{\tau_{0}+t}\left(\left(\tau_{0}+t\right)-y\right)^{\alpha} \int_{-\infty}^{\infty}\left|u^{\prime \prime}(x+i y, t)\right|^{2} d x d y \\
\leq & 2 \alpha \int_{0}^{\tau_{0}+t}\left(\left(\tau_{0}+t\right)-y\right)^{\alpha-1} \int_{-\infty}^{\infty}|u(x+i y, t)|^{2} d x d y \\
& +2 \int_{0}^{\tau_{0}+t}\left(\left(\tau_{0}+t\right)-y\right)^{\alpha} \int_{-\infty}^{\infty}|u(x+i y, t)|^{3} d x d y \\
= & I_{1}+I_{2} .
\end{aligned}
$$

For the linear term $I_{1}$, we need to consider two (possible) cases, $\left(\tau_{0}+t\right)-y$ $\geq 1$ and $\left(\tau_{0}+t\right)-y<1$. In the first case, $\left(\left(\tau_{0}+t\right)-y\right)^{\alpha-1} \leq\left(\left(\tau_{0}+t\right)-y\right)^{\alpha}$, and we can simply write

$$
I_{1} \leq 2 \alpha \int_{0}^{\tau_{0}+t}\left(\left(\tau_{0}+t\right)-y\right)^{\alpha} \int_{-\infty}^{\infty}|u(x+i y, t)|^{2} d x d y .
$$

In the second case, $\left(\left(\tau_{0}+t\right)-y\right)^{\alpha-1}>\left(\left(\tau_{0}+t\right)-y\right)^{\alpha}$, and we will have to use Proposition 1 (notice that the assumptions in the theorem imply $\alpha-1>-1$, and the proposition is applicable).

We restrict our considerations to $\left[0, T^{*}\right]$, where $T^{*}$ is as in (12), so that the $L^{2}$-norm of the trace of a complexified solution on the real axis is uniformly bounded. Also, the constants depending on the initial parameters $\tau_{0}, \alpha$, and $\left\|u_{0}\right\|_{B_{\alpha}^{2}\left(S_{\tau_{0}}\right)}$ will be denoted by a generic $K$. 
Adopting the above, Proposition 1 and scaling down the exponent imply

$$
\begin{aligned}
I_{1} & \leq K+K \int_{0}^{\tau_{0}+t}\left(\left(\tau_{0}+t\right)-y\right)^{\alpha+1} \int_{-\infty}^{\infty}\left|u^{\prime}(x+i y, t)\right|^{2} d x d y \\
& \leq K+K \int_{0}^{\tau_{0}+t}\left(\left(\tau_{0}+t\right)-y\right)^{\alpha} \int_{-\infty}^{\infty}\left|u^{\prime}(x+i y, t)\right|^{2} d x d y .
\end{aligned}
$$

By standard interpolation,

$$
\begin{aligned}
\int_{-\infty}^{\infty} \mid u^{\prime}(x & +i y, t)\left.\right|^{2} d x \\
\leq & \left(\int_{-\infty}^{\infty}|u(x+i y, t)|^{2} d x\right)^{1 / 2}\left(\int_{-\infty}^{\infty}\left|u^{\prime \prime}(x+i y, t)\right|^{2} d x\right)^{1 / 2},
\end{aligned}
$$

which in turn implies (by the Cauchy-Schwarz inequality in the variable $y$ )

$$
\begin{aligned}
\int_{0}^{\tau_{0}+t}\left(\left(\tau_{0}+t\right)\right. & -y)^{\alpha} \int_{-\infty}^{\infty}\left|u^{\prime}(x+i y, t)\right|^{2} d x d y \\
\leq & \left(\int_{0}^{\tau_{0}+t}\left(\left(\tau_{0}+t\right)-y\right)^{\alpha} \int_{-\infty}^{\infty}|u(x+i y, t)|^{2} d x d y\right)^{1 / 2} \\
& \times\left(\int_{0}^{\tau_{0}+t}\left(\left(\tau_{0}+t\right)-y\right)^{\alpha} \int_{-\infty}^{\infty}\left|u^{\prime \prime}(x+i y, t)\right|^{2} d x d y\right)^{1 / 2} .
\end{aligned}
$$

Inserting (20) in (18) and polarizing yields

$$
\begin{aligned}
I_{1} \leq & K+K \int_{0}^{\tau_{0}+t}\left(\left(\tau_{0}+t\right)-y\right)^{\alpha} \int_{-\infty}^{\infty}|u(x+i y, t)|^{2} d x d y \\
& +\frac{1}{2} \int_{0}^{\tau_{0}+t}\left(\left(\tau_{0}+t\right)-y\right)^{\alpha} \int_{-\infty}^{\infty}\left|u^{\prime \prime}(x+i y, t)\right|^{2} d x d y
\end{aligned}
$$

Notice that we can incorporate (17) in (21), and hence (21) is our final bound on $I_{1}$.

We now turn our attention to the nonlinear term $I_{2}$. The $x$-integral in $I_{2}$ is estimated as

$$
\int_{-\infty}^{\infty}|u(x+i y, t)|^{3} d x \leq\|u\|_{L^{\infty}\left(S_{\tau_{0}+t}\right)} \int_{-\infty}^{\infty}|u(x+i y, t)|^{2} d x
$$

where $\|u\|_{L^{\infty}\left(S_{\tau_{0}+t}\right)}$ can be bounded utilizing Proposition 2. That leads to 


$$
\begin{aligned}
I_{2} \leq & K \int_{0}^{\tau_{0}+t}\left(\left(\tau_{0}+t\right)-y\right)^{\alpha-1 / 2} \int_{-\infty}^{\infty}|u(x+i y, t)|^{2} d x d y \\
& +K\left(\int_{0}^{\tau_{0}+t}\left(\left(\tau_{0}+t\right)-y\right)^{\alpha} \int_{-\infty}^{\infty}|u(x+i y, t)|^{2} d x d y\right)^{1 / 4} \\
& \times\left(\int_{0}^{\tau_{0}+t}\left(\left(\tau_{0}+t\right)-y\right)^{\alpha} \int_{-\infty}^{\infty}\left|u^{\prime}(x+i y, t)\right|^{2} d x d y\right)^{1 / 4} \\
& \times \int_{0}^{\tau_{0}+t}\left(\left(\tau_{0}+t\right)-y\right)^{(\alpha-1) / 2} \int_{-\infty}^{\infty}|u(x+i y, t)|^{2} d x d y
\end{aligned}
$$

As for the linear term, we need to consider two cases, $\left(\tau_{0}+t\right)-y \geq 1$ and $\left(\tau_{0}+t\right)-y<1$. In the first case

$$
\begin{aligned}
I_{2} \leq & K \int_{0}^{\tau_{0}+t}\left(\left(\tau_{0}+t\right)-y\right)^{\alpha} \int_{-\infty}^{\infty}|u(x+i y, t)|^{2} d x d y \\
& +K\left(\int_{0}^{\tau_{0}+t}\left(\left(\tau_{0}+t\right)-y\right)^{\alpha} \int_{-\infty}^{\infty}|u(x+i y, t)|^{2} d x d y\right)^{5 / 4} \\
& \times\left(\int_{0}^{\tau_{0}+t}\left(\left(\tau_{0}+t\right)-y\right)^{\alpha} \int_{-\infty}^{\infty}\left|u^{\prime}(x+i y, t)\right|^{2} d x d y\right)^{1 / 4} .
\end{aligned}
$$

In the second case, we apply Proposition 1 to both terms (both exponents satisfy the assumptions in the proposition), and then scale down the exponents obtaining

$$
\begin{aligned}
I_{2} \leq & K+K \int_{0}^{\tau_{0}+t}\left(\left(\tau_{0}+t\right)-y\right)^{\alpha} \int_{-\infty}^{\infty}\left|u^{\prime}(x+i y, t)\right|^{2} d x d y \\
& +K\left(\int_{0}^{\tau_{0}+t}\left(\left(\tau_{0}+t\right)-y\right)^{\alpha} \int_{-\infty}^{\infty}|u(x+i y, t)|^{2} d x d y\right)^{1 / 4} \\
& \times\left(\int_{0}^{\tau_{0}+t}\left(\left(\tau_{0}+t\right)-y\right)^{\alpha} \int_{-\infty}^{\infty}\left|u^{\prime}(x+i y, t)\right|^{2} d x d y\right)^{1 / 4} \\
& +K\left(\int_{0}^{\tau_{0}+t}\left(\left(\tau_{0}+t\right)-y\right)^{\alpha} \int_{-\infty}^{\infty}|u(x+i y, t)|^{2} d x d y\right)^{1 / 4} \\
& \times\left(\int_{0}^{\tau_{0}+t}\left(\left(\tau_{0}+t\right)-y\right)^{\alpha} \int_{-\infty}^{\infty}\left|u^{\prime}(x+i y, t)\right|^{2} d x d y\right)^{5 / 4}
\end{aligned}
$$

Notice that to be able to scale down the exponent in the last term, we need $(\alpha+3) / 2 \geq \alpha$, which is the source of our restrictive assumption on $\alpha$. 
Collecting (24) and (25) gives

$$
\begin{aligned}
I_{2} \leq & K+K \int_{0}^{\tau_{0}+t}\left(\left(\tau_{0}+t\right)-y\right)^{\alpha} \int_{-\infty}^{\infty}|u(x+i y, t)|^{2} d x d y \\
& +K \int_{0}^{\tau_{0}+t}\left(\left(\tau_{0}+t\right)-y\right)^{\alpha} \int_{-\infty}^{\infty}\left|u^{\prime}(x+i y, t)\right|^{2} d x d y \\
& +K\left(\int_{0}^{\tau_{0}+t}\left(\left(\tau_{0}+t\right)-y\right)^{\alpha} \int_{-\infty}^{\infty}|u(x+i y, t)|^{2} d x d y\right)^{1 / 4} \\
& \times\left(\int_{0}^{\tau_{0}+t}\left(\left(\tau_{0}+t\right)-y\right)^{\alpha} \int_{-\infty}^{\infty}\left|u^{\prime}(x+i y, t)\right|^{2} d x d y\right)^{1 / 4} \\
& +K\left(\int_{0}^{\tau_{0}+t}\left(\left(\tau_{0}+t\right)-y\right)^{\alpha} \int_{-\infty}^{\infty}|u(x+i y, t)|^{2} d x d y\right)^{5 / 4} \\
& \times\left(\int_{0}^{\tau_{0}+t}\left(\left(\tau_{0}+t\right)-y\right)^{\alpha} \int_{-\infty}^{\infty}\left|u^{\prime}(x+i y, t)\right|^{2} d x d y\right)^{1 / 4} \\
& +K\left(\int_{0}^{\tau_{0}+t}\left(\left(\tau_{0}+t\right)-y\right)^{\alpha} \int_{-\infty}^{\infty}|u(x+i y, t)|^{2} d x d y\right)^{1 / 4} \\
& \times\left(\int_{0}^{\tau_{0}+t}\left(\left(\tau_{0}+t\right)-y\right)^{\alpha} \int_{-\infty}^{\infty}\left|u^{\prime}(x+i y, t)\right|^{2} d x d y\right)^{5 / 4} .
\end{aligned}
$$

Utilizing (20), and polarizing, we obtain our final bound on $I_{2}$ :

$$
\begin{aligned}
I_{2} \leq & K+K \int_{0}^{\tau_{0}+t}\left(\left(\tau_{0}+t\right)-y\right)^{\alpha} \int_{-\infty}^{\infty}|u(x+i y, t)|^{2} d x d y \\
& +K\left(\int_{0}^{\tau_{0}+t}\left(\left(\tau_{0}+t\right)-y\right)^{\alpha} \int_{-\infty}^{\infty}|u(x+i y, t)|^{2} d x d y\right)^{3 / 7} \\
& +K\left(\int_{0}^{\tau_{0}+t}\left(\left(\tau_{0}+t\right)-y\right)^{\alpha} \int_{-\infty}^{\infty}|u(x+i y, t)|^{2} d x d y\right)^{11 / 7} \\
& +K\left(\int_{0}^{\tau_{0}+t}\left(\left(\tau_{0}+t\right)-y\right)^{\alpha} \int_{-\infty}^{\infty}|u(x+i y, t)|^{2} d x d y\right)^{7 / 3} \\
& +\int_{0}^{\tau_{0}+t}\left(\left(\tau_{0}+t\right)-y\right)^{\alpha} \int_{-\infty}^{\infty}\left|u^{\prime \prime}(x+i y, t)\right|^{2} d x d y
\end{aligned}
$$


Inserting (21) and (27) in the differential inequality (16) yields

$$
\begin{aligned}
\frac{d}{d t} \int_{0}^{\tau_{0}+t}\left(\left(\tau_{0}+t\right)-y\right)^{\alpha} \int_{-\infty}^{\infty}|u(x+i y, t)|^{2} d x d y \\
\leq K+K \int_{0}^{\tau_{0}+t}\left(\left(\tau_{0}+t\right)-y\right)^{\alpha} \int_{-\infty}^{\infty}|u(x+i y, t)|^{2} d x d y \\
+K\left(\int_{0}^{\tau_{0}+t}\left(\left(\tau_{0}+t\right)-y\right)^{\alpha} \int_{-\infty}^{\infty}|u(x+i y, t)|^{2} d x d y\right)^{3 / 7} \\
+K\left(\int_{0}^{\tau_{0}+t}\left(\left(\tau_{0}+t\right)-y\right)^{\alpha} \int_{-\infty}^{\infty}|u(x+i y, t)|^{2} d x d y\right)^{11 / 7} \\
+K\left(\int_{0}^{\tau_{0}+t}\left(\left(\tau_{0}+t\right)-y\right)^{\alpha} \int_{-\infty}^{\infty}|u(x+i y, t)|^{2} d x d y\right)^{7 / 3}
\end{aligned}
$$

or, equivalently,

$$
\frac{d}{d t} \varphi \leq K+K \varphi^{3 / 7}+K \varphi+K \varphi^{11 / 7}+K \varphi^{7 / 3} .
$$

Solving the differential inequality (29) finishes the proof.

REMARK 4 . The estimates in the proof are formal a priori estimates that can be made rigorous by considering a sequence of linear approximations [GK1, GK2].

REMARK 5. An immediate consequence of Theorem 1 is that (10) can "push away" an algebraic-type singularity, provided the order of the singularity is small enough $(\alpha \leq 3)$. This constitutes a very strong smoothing property, and a restriction on $\alpha$ is expected. So, loss of analyticity (and hence, a finite-time blow-up) can be caused only by "strong enough" complex singularities that a priori cannot be repelled.

REMARK 6. It should be noted that, in general, the migration of complex singularities to the real axis is not the only scenario through which the loss of analyticity may occur. Some "more discontinuous" scenarios are possible, as well as uniform-in-space growth of a solution.

\section{References}

[CEES] P. Collet, J.-P. Eckmann, H. Epstein and J. Stubbe, Analyticity for the KuramotoSivashinsky equation, Phys. D, 67 (1993), 321-326.

[D] P. L. Duren, Theory of $H^{p}$ Spaces, Academic Press, New York, 1970.

[FeTi] A. B. Ferrari and E. S. Titi, Gevrey regularity for nonlinear analytic parabolic equations, Comm. Partial Differential Equations 23 (1998), 1-16. 
[FT] C. Foiaş and R. Temam, Gevrey class regularity for the solutions of the NavierStokes equations, J. Funct. Anal. 87 (1989), 359-369.

[GK1] Z. Grujić and I. Kukavica, Space analyticity for the Navier-Stokes and related equations with initial data in $L^{p}$, J. Funct. Anal. 152 (1998), 447-466.

[GK2] - - - Space analyticity for the nonlinear heat equation in a bounded domain, J. Differential Equations 154 (1999), 42-54.

[NOW] A. Nakamura, F. Ohya, and H. Watanabe, On some properties of weighted Bergman spaces, Proc. Fac. Sci. Tokai Univ. 15 (1979), 33-44.

Department of Mathematics

Department of Mathematics

University of Texas

Austin, TX 78712, U.S.A.

University of Virginia

E-mail: grujic@math.utexas.edu

Received November 6, 2000

Revised version March 14, 2001 